\title{
Synthesis of Patterned AIN Nanoarray and its Photoluminance Property Investigation
}

\author{
Ming-zhe $\mathrm{Hu}^{\mathrm{a1}}$, Zhi-wei Zeng ${ }^{\mathrm{b1}}$, Yue Yin $^{\mathrm{c} 1}$ and Deng-hui $\mathrm{Ji}^{\mathrm{d1}, *}$ \\ ${ }^{1}$ Department of Physics and Electronics, Liupanshui Normal University, Liupanshui, Guizhou \\ 553004, China \\ diidenghui2007@163.com \\ ${ }^{*}$ Corresponding author
}

Keywords: AIN nanoarray Formatting, Photoluminance property, Microstructure, CVD.

\begin{abstract}
Chemical vapor deposition (CVD) method is employed in the present paper to grow patterned Aluminium nitride (AlN) nanowire array. The AlN nanoarray is grown on Si substrate through patterned polystyrene spheres (PS) colloid template. The microstructure of AIN nanoarray is investigated by SEM, XRD and Photoluminescence (PL) spectrum. The results show that the full width at half-maximum (FWHM) of the XRD diffraction peaks of AlN nanostructure are very narrow, which means the good crystalline structure of the AIN nanowire. From the SEM photos, the cyclical morphology of the AlN nanostructure can be observed. The cycle is equal to the diameter of the PS template. The PL spectrum of the patterned AlN indicates that a strong and narrow band of $3.8 \mathrm{eV}$ is emitted in the patterned AlN sample, which is due to the nitrogen vacancies in AlN.
\end{abstract}

\section{Introduction}

AlN is a hexagonal wurtzite structure III-IV compound, which has the largest band gap in III nitrides. Dimension effect makes it special physical properties, which is considered a very promising material for semiconductor devices. AIN has high thermal conductivity and breakdown voltage, at the same time, it also has many other advantages, such as high mechanical strength, good thermal expansion coefficient, large surface acoustic propagation speed, relatively high piezoelectric constant and very low electron affinity. Possessing a negative electron affinity means that electrons excited into the conduction band can be freely emitted into vacuum.Therefore, AlN is suitable for field emission display (FED) application. Actually, at present, the factor impeding the big scale production of FED is the anode material and its array production. Even distribution, high lightening and easy production anode nanoarray continuously becomes the key points of worldwide researchers. The III-IV compound AIN is an ideal anode emitting candidate due to the above advantages. Especially, its one dimensional nanoarray structure, possesses the capability of significantly enhancing the localized electrical field, lowering the opening field emission voltage and increasing field emission current, which makes it a great potential usage in FED microelectronic industry and the growth of one dimensional semiconductor nanostructures with proper density and defect free has been especially focused on by researchers in nanotechnology.

In an inert atmosphere, AlN is stable in high temperature. In the air, when the temperature is higher than $700 \circ \mathrm{C}$, it will be oxidation. In addition, when the temperature is higher than $1000 \mathrm{oC}$, the AlN can still maintain its mechanical properties and piezoelectric properties. Therefore, AlN possesses a widespread application, especially for its nanostructured materials. Its special chemical and physical properties arouse much worldwide research. AlN nanostructures can be synthesized via various methods, such as CVD method [1-2], arc discharge method [3-4], physical vapor deposition (PVD) method [5] and so on. These nanostructures include nanowhisker, nanowire, nanotip, nanorod, nanocone, nanocrater, nanoneedle, nanotube, nanoring, nanobrush and nanocomb. For example, Li et al. [6] synthesized various one-dimensional AlN nanoarrays by adjusting the substrates and the temperature in the CVD process. Yu et al. [7-8] reported the fabrication of different structures of AIN nanoflowers using CVD method. The PL spectra of these nanoflowers exhibited different blue bands, 
which located at $437 \mathrm{~nm}$ and $488 \mathrm{~nm}$, respectively. Chen et al. [9] synthetized AlN nanowhiskers consisted of nanotips growing out from the surfaces of sphere-like cores. The PL of the nanostructures were measured with a Xe lamp as light source and a broad blue emission band ranging from ultraviolet $(416 \mathrm{~nm})$ to yellow $(564 \mathrm{~nm})$ was observed from the PL spectra. Zhonglin Wang et al.[10] reported the growth of well-aligned AIN nanorods with hairy surfaces by a vapor-solid (VS) process. The well-aligned AIN nanorods with hairy surfaces can not only provide a new hierarchical nanostructure but also serve as a promising candidate for FE emitters because of their low electron affinity and the geometry of the multiple-nanotip surfaces. The as-prepared AlN sample had a low turn-on field of $3.8 \mathrm{~V} \mu \mathrm{m}-1$ and an intensive light emission at 519nm. Y. K. Byeun et al.[11] reported the controlled growth by HVPE of one dimensional single-crystalline AIN nanostructure arrays by employing various controlled growth conditions, such as growth temperature, gas flow rate, and the effects of the use or non-use of catalysts, and they also investigated the field emission properties of these single-crystalline AIN nanostructures, the results showed that the as-grown AlN nanostructures had a low turn-on field and threshold field of 2.25 and $3.58 \mathrm{Vmm}-1$, respectively. However, the randomly distributed AIN nanostructure is not suitable for the final FED anode due to the screening effect, only the highly ordered and orientated AlN nanoarray is the key for production even emission, high lightening, low loss and addressable FED devices. Therefore, in the present paper, we employ the CVD method to prepare the highly order AIN nanostructure by employing patterned template.

\section{Experiment}

In this experiment, polystyrene spheres were prepared by introducing into the reaction flask a solution containing PVP (MW 10000, $2 \mathrm{~g}, 1.8 \% \mathrm{w} / \mathrm{v}$ of total solution, ACS reagent grade) dissolved in a mixture of ethanol and DI water. The temperature of the mechanically stirred solution was maintained at $65 \mathrm{oC}$. Nitrogen was used to exclude air during the polymerization period. Another solution containing the initiator benzyl peroxide $(1.3 \mathrm{~g}, 1.2 \% \mathrm{w} / \mathrm{v}$ of total solution, ACS reagent grade) and styrene $(6.2 \mathrm{ml}, 5.2 \% \mathrm{w} / \mathrm{v}$ of total solution, ACS reagent grade) was then added to the reaction flask. The polymerization reaction was continued for $24 \mathrm{~h}$ and the flask was then cooled. The spheres formed were washed with ethanol and then water. The monolayer of latex spheres was created. After removing the polystyrene spheres, ordered and patterned pores was left on the silicon.

The synthesis of patterned AlN nanowires was carried out in a horizontal two-temperature zone tubular furnace. $\mathrm{Si}(111)$ was pretreated by the above method, and then silicon was served as substrate placed at the center of the high temperature zone, AlCl3 powder (chemical purity $99.99 \%$ ) was used as aluminum source and ammonia was employed as nitrogen resource. About 8 gram $\mathrm{AlCl} 3$ is placed in an alumina boat, and the boat was put in the upstream place about one boat away from the $\mathrm{Si}$ substrate. The system was flushed with $\mathrm{NH} 3 / \mathrm{N} 2$ mixed gas about an hour to remove oxygen and moisture. The furnace was heated under a NH3/N2 flow of $20 \mathrm{sccm}$ at $15 \mathrm{oC} / \mathrm{min}$, and the temperature was $830 \mathrm{oC}$. The system was maintained at constant temperature and gas flow for $2 \mathrm{hrs}$. After that, the system was cooled down to room temperature under $100 \mathrm{sccm} \mathrm{NH} 3 / \mathrm{N} 2$.

The as-prepared products were characterized by scanning electron microscopy (SEM, model Gemini LEO 1530 with energy-dispersive X-ray spectroscopy installed), X-ray diffraction (XRD, model Bruker D8 Advance, $\lambda=1.54056 \AA$ ) with $\mathrm{Cu} \mathrm{K} \alpha$ radiation. The PL spectra were recorded by exciting with the $200 \mathrm{~nm}$ Xe laser.

\section{Results and Discussion}

Figure 1(a) shows the self-assembled polystyrene colloidal template, Fig. 1(b) shows the patterned $\mathrm{SiO} 2$ and Fig. 1(c-d) illustrates the morphology of the patterned AlN nanoflowers. In the experiment, the diameter of the polystyrene spheres is about $860 \mathrm{~nm}$, it's approximately equal to growth cycle of the AIN nanoflowers. So it can be concluded that the AIN nanostructure is almost grown on the exposed place on PS template decorated Si substrate. In addition, the nanoflowers are dispersed from the middle to both sides, in other words, the nanoflowers are not grown on the wall of the template. 


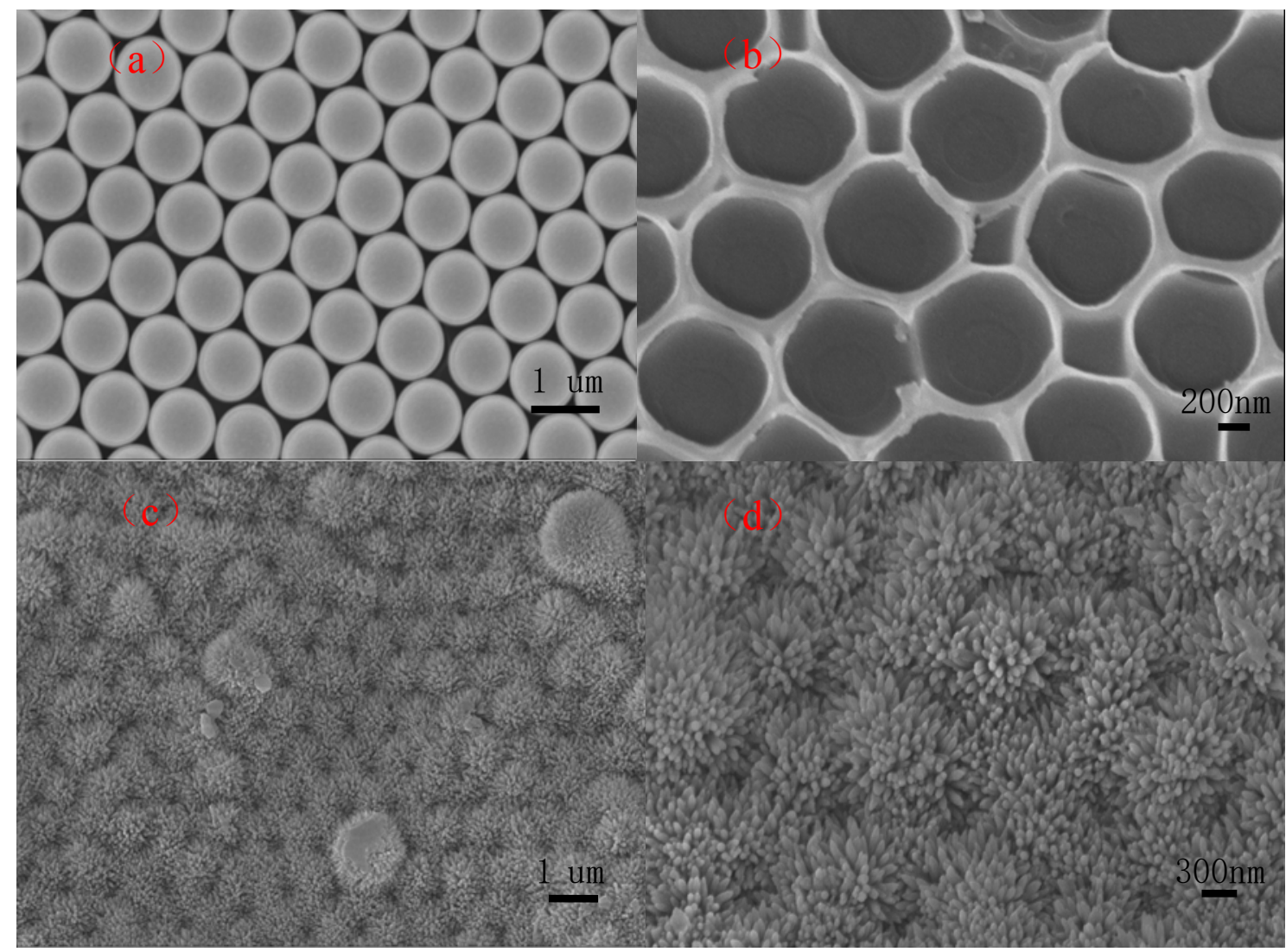

Figure 1(a) The morphology of monolayer polystyrene colloidal,(b)The morphology of the template without polystyrene spheres, (c) and(d) The morphology of the patterned AlN nanoflowers

The XRD pattern of the as-prepared AIN nanostructure is illustrated in Fig.2. As can be seen, the patterned AlN sample exhibits pure wurtzite structure, according to the standard JCPDS card No. 041542. The 2 Theta angles of the diffraction peaks are located at $35.9^{\circ}, 37.8^{\circ}$ and $59.2^{\circ}$, which are correspond to (002), (101) and (110) planes of AlN wurtzite structure, respectively. Other peaks are corresponding to alumina phase. The generation of alumina phase results from the excess oxygen in the furnace during the AlN growth. In addition, a large slope appears in the initial 2Theta angle in the figure, which can be attributed to the silicon substrate. Because compared with the $\mathrm{Si}(111)$ substrate, there is much less AlN sample content, the signal of the substrate is thus much stronger than the AlN sample. What's more, the FWHM of the diffraction peaks are very narrow, which means the crystalline structure of the AIN sample is good.

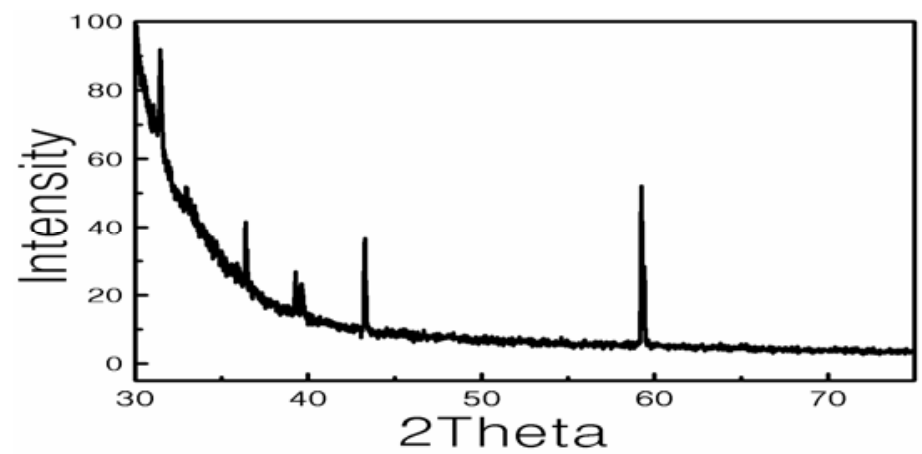

Figure 2. XRD pattern of the as-synthesized AlN nanostructures

The PL spectrum of the as-prepared AIN sample collected at room temperature is indicated in Fig.3. Irradiation of AlN sample by UV light results in formation of a strong PL spectrum, in which the 
$3.65 \mathrm{eV}$ band is predominant within the UV region. Youngman et al [12] proposed that a broad band at $2.7(459 \mathrm{~nm})$ and $3.8 \mathrm{eV}(326 \mathrm{~nm})$ from AlN single crystals grown under nitrogen-deficient conditions results from the vacancy of $\mathrm{N}$, but without giving detailed information. In our experiment, the growth temperature is relative low, and there also might be oxygen residue in the furnace during AlN growth, thus, there is a large number of nitrogen vacancies in the as-prepared AlN sample. We can see that the PL intensity of the nanostructure is strong, and the FWHM is narrow, it means that less impurity incorporated into the sample.

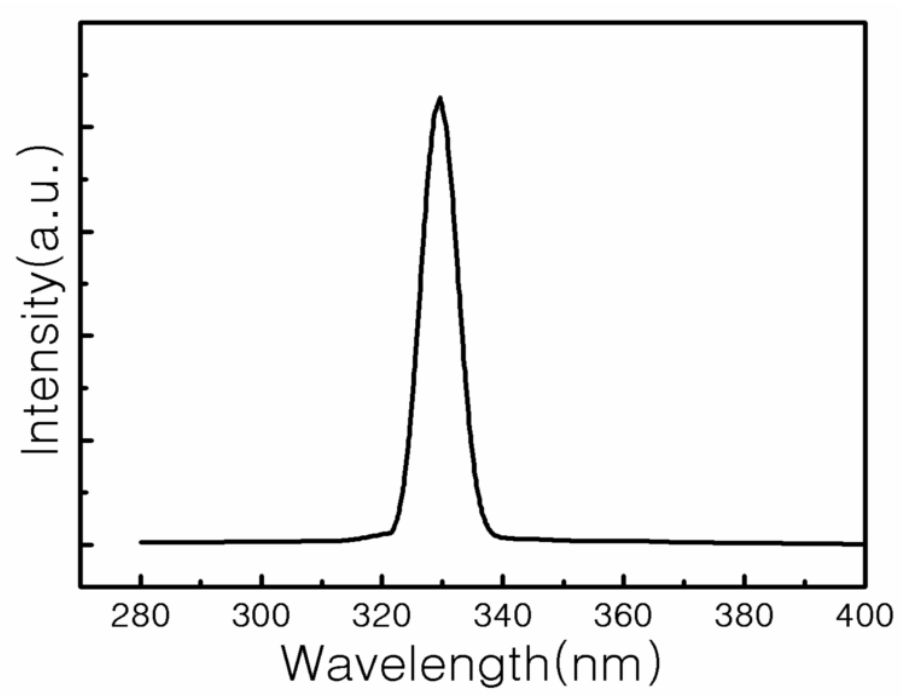

Figure 3. The Photoluminescence spectra of AlN nanostructures

\section{Conclusions}

We have prepared patterned AIN nanoflowers using chemical vapor deposition(CVD) method. The as-prepared products were characterized by SEM, XRD, and PL, the PL spectra were recorded by exciting with the $200 \mathrm{~nm}$ Xe laser. the crystalline structure of the AlN sample is good and less impurity incorporated into the sample.

\section{Acknowledgment}

This work is supported by the Natural Science Foundation of China (No.61076049, 51567017), the Physical Electronic Key Discipline of Guizhou Province No. ZDXK201535, the Outstanding Young Scientist Cultivation Program of Guizhou Province No.201522, the Joint Science Foundation of Liupanshui City and Guizhou Province No. LH20147448 and LH20147449, the Key Laboratory of Opt-electrical Information Technology of Liupanshui City (No.52020201420205), the MIT Creative Team of Liupanshui Normal University No.LPSSYKJTD201402, the Recruitment Program of Liupanshui Normal University No. LPSSYKYJJ201403 and LPSSYKYJJ201404, the Recruitment Program of Guizhou University No.2012032, the Key Project of the Education Department of Guizhou Province (Nos.2013174 and 2015379).

\section{References}

[1] J. Yang ,T. W. Liu, C. W. Hsu. et al. Nanotechnology, 2006,17, S321-S326

[2] J. Zheng, Y. Yang, B. Yu. et al. ACS nano, 2008, 2, 134-142

[3] W. W. Lei, D. Liu, P. W. Zhu. et al. J. Phys. Chem. C 2008, 112, 13353-13358

[4] W. W. Lei, D. Liu, J. Zhang. et al. Crystal Growth \& Design, 2009, 9, 1489-1493 
[5] Q. Zhao, J. Xu, X. Y. Xu. et al. Appl. Phys. Lett.2004,85, 5331-5333

[6] J. Zheng,Y. Yang, B. Yu, X.b. Song, X.G. Li, ACS Nano. 2, 134-142 (2008)

[7] L.S. Yu, Z. Hu, Y.W. Ma, K.F. Huo, Y. Chen, H. Sang, W.W. L, Y.N. Lu, Diamond \& Related Materials. 16, 1636-1642 (2007)

[8] L.S. Yu, N. Liu, C.Y. He, Q. Wu, Z. Hu, J. Phys. Chem. C. 113, 14245-14248 (2009)

[9] H.T. Chen, X.L. Wu, X. Xiong, W.C. Zhang, L.L. Xu, J. Zhu, P.K. Chu, J. Phys. D: Appl. Phys. 41, $025101(2008)$

[10] J. H. He, R. S. Yang, Y. L. Chueh, L. J. Chou, L. J. Chen, Z. L. Wang, Adv. Mater., 18, 650-654(2008)

[11] Y.K. Byeun, R. Telle, S.H. Jung, S.C. Choi, H.I. Hwang, Chem. Vapor Disposition, 16, 72-79 (2010)

[12] R. A. Youngman, J. H. Harris, D. A. Chernoff. Ceram.Trans. 1989,5,309-312 